\title{
Acute Effect of Auricular Vagus Nerve Stimulation on Cycle Ergometer Test and Physiological Parameters in Healthy Young Individuals: A Pilot Study \\ Sefa Haktan HATIK, PT, MSc, PhD ${ }^{1^{*}}$ (D), Berkay Eren Pehlivanoğlu, PT, MSc, PhD ${ }^{2}$ (D), Mesut ARSLAN, PT, MSc, PhD ${ }^{3}$, Çilem TAŞKIN, MSc ${ }^{4}$ (D) and Ali Veysel ÖZDEN, MD ${ }^{5}$ (iD

${ }^{1}$ Graduate School, Bahçeşehir University, Turkey

${ }^{2}$ Department of Therapy and Rehabilitation, Vocational School of Health Services, Rumeli University, Turkey

${ }^{3}$ Head of Therapy and Rehabilitation Department, Vocational School of Health Services, Gümüşhane University, Turkey

${ }^{4}$ Department of Health Care Services, Türkeli Vocational School Sinop University, Turkey

${ }^{5}$ Physiotherapy and Rehabilitation Department, Health Sciences Faculty, Bahçeşehir University, Turkey

*Corresponding author: Sefa Haktan HATIK, PT, Msc, PhD(c), Graduate School, Bahçeşehir University, Turkey, Tel: +905058761553

\begin{abstract}
Background: This study is to investigate the effect of non-invasive auricular VNS on cycle ergometer test and physiological parameters in healthy individuals.

Methods: 46 people participated in the study. The participants were randomly divided into 3 groups as Above Threshold, Under Threshold and Control according to the sensation of electrical current on ears. The participants were evaluated 3 times; before the application, after the first and second bicycle exercises. Numerical pain scale, pulse rate, blood pressure, respiratory rate, and distance travelled during exercise for cycle ergometer test.

Results: There was no significant difference in $1^{\text {st }}$ measurement, $2^{\text {nd }}$ measurement, $3^{\text {rd }}$ measurement of pulse rate, saturation value and performance in the control group, sub-threshold group and above threshold group $(p>0.05)$. The $2^{\text {nd }}$ measurement of NPS value was significantly lower in the above threshold group than in the control group and the below threshold group $(p<0.05)$. The $1^{\text {st }}$ measurement, $2^{\text {nd }}$ measurement, $3^{\text {rd }}$ measurement respiratory rate and $3^{\text {rd }}$ measurement diastolic pressure values were significantly higher in the sub-threshold group $(p<0.05)$.

Conclusion: In healthy individuals, short-term non-invasive auricular VNS does not affect cycle ergometer test during exercise. This may be due to the fact that the application time is kept short and the application is a single time. In VNS application, feeling the current and/or current intensity may affect parameters such as respiratory rate and blood pressure.
\end{abstract}

\section{Keywords}

Vagus nerve stimulation, Physiological parameters, Cycle ergometer test

\section{Introduction}

The autonomic nervous system is the system that controls a significant part of the visceral functions of the body. Efferent autonomic signals are transmitted to the body through sympathetic and parasympathetic nerves. Parasympathetic fibers originate from 3, 7, $9,10^{\text {th }}$, cranial nerves and sacral spinal nerve roots. Approximately 75 percent of all parasympathetic fibers circulate within the vagus ( $10^{\text {th }}$ cranial nerve) nerve and distribute to the visceral organs [1].

Hypertension, smoking, diabetes, physical and emotional stress reduce vagal activity and thus the effectiveness of the parasympathetic nervous system. Many diseases can be prevented by controlling vagal activity [1]. Vagus nerve is responsible for the regulation of inflammation processes in chronic diseases $[2,3]$. At the same time, vagal activity control is of great importance in improving cardiac functions $[4,5]$. In addition, people with strong vagal activity are more likely to recover faster after injury or disease [6]. 
Many applications have been developed through the vagus nerve to increase parasympathetic activity [1]. In 1990, invasive vagus nerve stimulation was first performed in treatment-resistant epilepsy patients and successful results were obtained [7]. However, various complications such as tissue injuries, hematoma, infection, cough, sore throat, hoarseness, dyspnea and chest pain may occur after invasive [1]. Noninvasive VNS methods have been implemented to avoid complications of invasive VNS, to increase the safety and tolerability of VNS and to provide more accessible and wide range of ease of use [8]. Non-invasive VNS has 2 types of administration as auricular and cervical stimulation [9].

Non-invasive auricular VNS was approved for treatment of epilepsy and depression in Europe in 2010 and pain in 2012 [9]. In randomized controlled studies in migraine patients, non-invasive VNS has been found to be effective and safe in the treatment of migraines [10-13]. In a double-blind randomized controlled study in healthy individuals, an increase in mechanical and pressure pain threshold and a decrease in mechanical pain sensitivity were found. In addition, no changes in cardiac and respiratory parameters were observed in the same study [14]. In a single-blind randomized controlled study in healthy individuals, non-invasive VNS was found to have a modulating effect on structures such as the hypothalamus, spinal trigeminal nucleus, pontine nucleus and parahippocampal gyrus [15]. In another study conducted on healthy individuals, noninvasive auricular VNS was found to increase creativity performance in different thinking [16]. In a study in rats, non-invasive VNS used in the treatment of trigeminal allodynia did not result in significant changes in blood pressure and pulse parameters [17].

\section{Materials and Methods}

\section{Participants}

This study included 46 individuals aged $18-35$ years in the home patient care laboratory at Sinop University Vocational School of Turkeli. Priorities were explained and the permission of the individuals was obtained. The number of females and males was divided into 3 groups as randomized. Participants were evaluated by Numerical Pain Scale (NPS), pulse rate, blood pressure, respiratory rate, and saturation measurement. In addition, the distance travelled during the exercise for sportive performance was recorded in kilometers. In the study, evaluation was conducted 3 times before the application, after the first and second bicycle exercises.

\section{Methods}

The study was double-blind randomized. Participants in the whole group were asked to perform bicycle exercise with maximum performance under 50 watts for 5 minutes. In all groups Vagustim device (Figure 1 and Figure 2) was used for stimulation. In the above

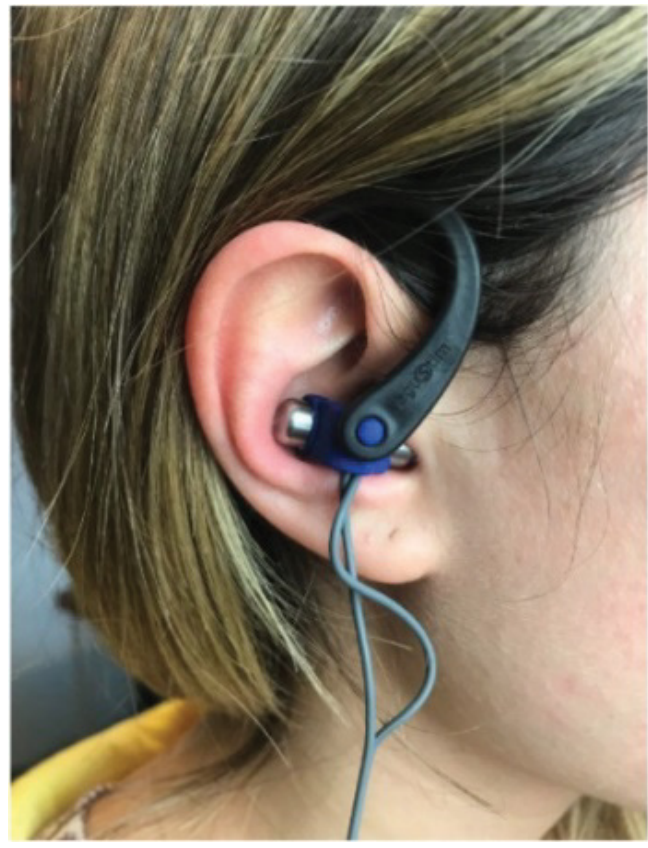

Figure 1: Vagustim device.

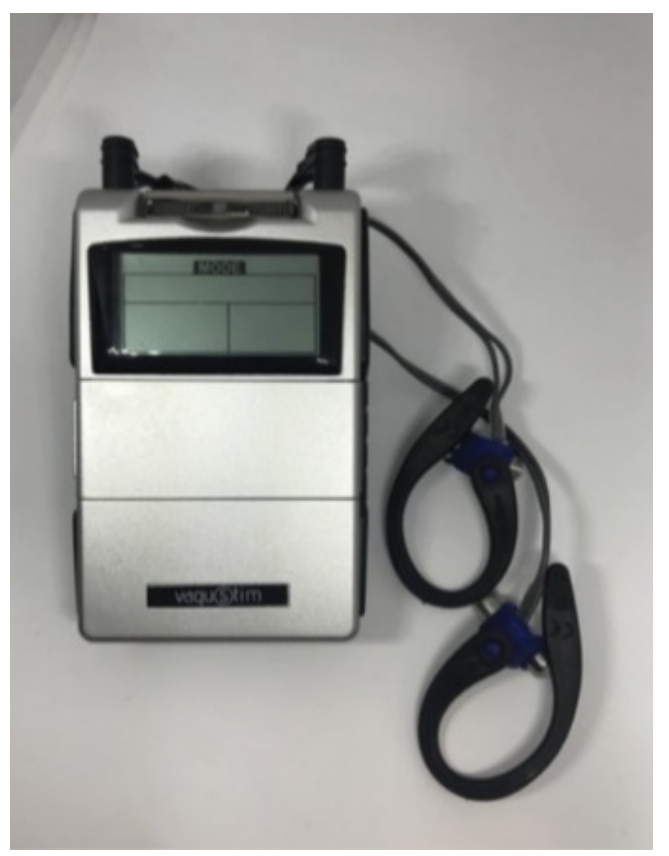

Figure 2: Vagustim device.

threshold group, biphasic current was applied as follows; frequency $10 \mathrm{~Hz}$, in Modulation mode (Modulation mode is a combination of pulse rate and pulse width modulation. The pulse rate and width are automatically varied in a cycle pattern. The pulse width is reduced by $50 \%$ from its original setting in 0.5 second, then the pulse rate is reduced by $50 \%$ from its original setting in 0.5 second. Total cycle time is 1 second.), the pulse width was $300 \mu \mathrm{s}$. The current intensity was kept constant where the participant felt the current comfortably and applied for 5 minutes. VNS was applied bilaterally to transmit current from the tragus and concha parts of the ear. After the first bicycle exercise, the participants were re-evaluated. In the second bicycle exercise, it was 
Table 1: Demographic information.

\begin{tabular}{|c|c|c|c|c|}
\hline & & Min.-Max. & Median & Mean. $\pm \mathrm{sd} / \mathrm{n}-\%$ \\
\hline \multicolumn{2}{|l|}{ Age } & $18.0-27.0$ & 19.0 & $19.2 \pm 1.5$ \\
\hline \multirow[t]{2}{*}{ Sex } & Female & & & $31-67.4 \%$ \\
\hline & Male & & & $15-32.6 \%$ \\
\hline \multicolumn{2}{|l|}{ BMI } & 16.9-36.1 & 22.4 & $22.9 \pm 3.7$ \\
\hline \multirow[t]{2}{*}{ Smoking } & $(-)$ & & & $29-63.0 \%$ \\
\hline & $(+)$ & & & $17-37.0 \%$ \\
\hline \multicolumn{2}{|c|}{ Cigarette (Each/ Day) } & $0.0-20.0$ & 0.0 & $3.9 \pm 6.2$ \\
\hline
\end{tabular}

Values are numbers or mean \pm standard deviation or number. Min: Minimum, Max:Maximum, Sd: Standard Deviation

asked to perform for 5 minutes under the same load without any application. The study was evaluated for the third time and the study was completed.

In the subthreshold group, the parameters were the same but the current was reduced to where the participant did not feel the current after the threshold value was reached and again applied for 5 minutes. It was re-evaluated after the first bicycle exercise. In the second bicycle exercise, he was asked to perform bicycle exercise with maximum performance for 5 minutes under the same load without any application. The study was evaluated for the third time and the study was completed.

In the control group, bicycle exercise was performed under the same load with the current-free headset produced for sham applications for 5 minutes. Participants were shown that the device was working, but no current was given. It was re-evaluated after the first bicycle exercise. In the second bicycle exercise, the device was removed and asked to perform bicycle exercise with maximum performance for 5 minutes under the same load. The study was evaluated for the third time and the study was completed.

\section{Statistical analysis}

Mean, standard deviation, median lowest, highest, frequency and ratio values were used in the descriptive statistics of the data. The distribution of the variables was measured by the Kolmogorovsimirnov test. Kruskalwallis, mann-whitney $u$ test was used in the analysis of quantitative independent data. Chi-squared test was used for the analysis of qualitative independent data. SPSS 26.0 program was used in the analyses (Table 1).

\section{Ethics}

The study complies with the Helsinki Declaration Principles and the ethical committee approval was obtained by the Scientific Research and Publication Ethics Committee of Gümüşhane University on 08.01.2020 with the decision no. 2020/01. The trial was registered with the ClinicalTrials.gov registry (Study Identifier: NCT04768738).

\section{Results}

The age and gender distribution of the patients in the control group, the sub-threshold group and the over-the-threshold group did not differ significantly ( $p$ $>0.05)$. The BMI value did not differ significantly $(p>$ 0.05 ) in the control group, the sub-threshold group and the over-the-threshold group. Smoking rate and cigarette package did not differ significantly $(p>0.05)$ in the control group, the sub-threshold group and the over-the-threshold group (Table 2).

In the control group, the sub-threshold group, the $1^{\text {st }}$ measurement and the $3^{\text {rd }}$ measurement NPS value did not differ significantly $(p>0.05)$. The $2^{\text {nd }}$ measurement NAS value was significantly lower in the above threshold group than in the control group and the below threshold group $(p<0.05)$. The $2^{\text {nd }}$ measurement NAS value did not differ significantly between the control group and the sub-threshold group $(p>0.05)$ (Table 2).

There was no significant difference $(p>0.05)$ in the control group, the sub-threshold group, the over-thethreshold group, according to the $1^{\text {st }}$ measurement, the $2^{\text {nd }}$ measurement, and the $3^{\text {rd }}$ measurement pulse value (Table 2).

The $1^{\text {st }}$ measurement, the $2^{\text {nd }}$ measurement, the $3^{\text {rd }}$ measurement respiratory rate were significantly higher in the sub-threshold group than the control group and the above threshold group $(p<0.05)$. There was no significant difference $(p>0.05)$ between the control group and the over-the-threshold group in terms of $1^{\text {st }}$ measurement, $2^{\text {nd }}$ measurement and $3^{\text {rd }}$ measurement respiration rate (Table 2 ).

In the control group, the $1^{\text {st }}$ measurement systolic pressure was significantly lower than the subthreshold and above threshold groups $(p<0.05)$. The $1^{\text {st }}$ measurement systolic pressure did not differ significantly $(p>0.05)$ between the sub-threshold and above threshold groups. The $2^{\text {nd }}$ measurement and the3rd measurement systolic pressure values did not differ significantly $(p>0.05)$ in the control group, the sub-threshold group and the over-the-threshold group (Table 2).

The $1^{\text {st }}$ measurement and the $2^{\text {nd }}$ measurement diastolic pressure values did not differ significantly $(p$ $>0.05$ ) in the control group, the sub-threshold group and the over-the-threshold group. In the sub-threshold 
Table 2: Comparison between Groups.

\begin{tabular}{|c|c|c|c|c|c|c|c|c|c|}
\hline & & \multicolumn{2}{|l|}{ Control Group } & \multicolumn{2}{|c|}{ Under Threshold Group } & \multicolumn{2}{|c|}{ Above Threshold Group } & \multicolumn{2}{|c|}{$P$ - Value } \\
\hline & & Mean. \pm sd/n-\% & Med & Mean. \pm sd/n-\% & Med & Mean $\pm s d / n-\%$ & Med & & \\
\hline \multicolumn{2}{|l|}{ AGE } & $19.8 \pm 2.1$ & 20 & $19.3 \pm 1.1$ & 19 & $18.7 \pm 0.5$ & 19 & 0.059 & $\mathrm{~K}$ \\
\hline \multirow[t]{2}{*}{ Sex } & Female & $11-68.8 \%$ & & $10-66.7 \%$ & & $10-66.7 \%$ & & \multirow[t]{2}{*}{0.990} & \multirow[t]{2}{*}{$\mathrm{X}^{2}$} \\
\hline & Male & $5-31.3 \%$ & & $5-33.3 \%$ & & $5-33.3 \%$ & & & \\
\hline \multicolumn{2}{|l|}{ BMI } & $22.8 \pm 3.3$ & 22 & $22.5 \pm 4.4$ & 22 & $23.3 \pm 3.6$ & 25 & 0.192 & K \\
\hline \multirow[t]{2}{*}{ Smoking } & $(-)$ & $8-50.0 \%$ & & $12-80.0 \%$ & & $9-60.0 \%$ & & \multirow[t]{2}{*}{0.214} & \multirow[t]{2}{*}{$\mathrm{X}^{2}$} \\
\hline & $(+)$ & $8-50.0 \%$ & & $3-20.0 \%$ & & $6-40.0 \%$ & & & \\
\hline \multicolumn{2}{|c|}{ Cigarette (Each/ Day) } & $4.56 \pm 5.44$ & 2 & $1.40 \pm 3.07$ & 0 & $5.60 \pm 8.45$ & 0 & 0.606 & K \\
\hline \multicolumn{10}{|l|}{ NPS } \\
\hline \multicolumn{2}{|l|}{$1^{\text {st }}$ measure } & $1.25 \pm 2.08$ & 0 & $0.53 \pm 1.13$ & 0 & $0.20 \pm 0.77$ & 0 & 0.198 & $\mathrm{~K}$ \\
\hline \multicolumn{2}{|l|}{$2^{\text {nd }}$ measure } & $1.75 \pm 2.11$ & 1 & $2.13 \pm 2.00$ & 2 & $0.40 \pm 0.91$ & 0 & 0.034 & $\mathrm{~K}$ \\
\hline \multicolumn{2}{|l|}{$3^{\text {th }}$ measure } & $1.56 \pm 2.00$ & 0 & $2.00 \pm 1.73$ & 2 & $0.67 \pm 1.05$ & 0 & 0.092 & $\mathrm{~K}$ \\
\hline \multicolumn{10}{|l|}{ Pulse } \\
\hline \multicolumn{2}{|l|}{$1^{\text {st }}$ measure } & $86.6 \pm 13.1$ & 87 & $83.6 \pm 11.5$ & 85 & $88.3 \pm 11.6$ & 86 & 0.569 & $\mathrm{~K}$ \\
\hline \multicolumn{2}{|l|}{$2^{\text {nd }}$ measure } & $106.4 \pm 18.6$ & 102 & $121.6 \pm 19.8$ & 123 & $106.5 \pm 20.4$ & 103 & 0.079 & $\mathrm{~K}$ \\
\hline \multicolumn{2}{|l|}{$3^{\text {th }}$ measure } & $113.4 \pm 18.8$ & 107 & $124.9 \pm 20.6$ & 122 & $110.5 \pm 22.9$ & 116 & 0.180 & $\mathrm{~K}$ \\
\hline \multicolumn{10}{|c|}{ Respiratory Rate } \\
\hline \multicolumn{2}{|c|}{$1^{\text {st }}$ measure } & $19.4 \pm 2.3$ & 19 & $21.6 \pm 2.1$ & 22 & $19.3 \pm 2.3$ & 20 & 0.016 & $\mathrm{~K}$ \\
\hline \multicolumn{2}{|l|}{$2^{\text {nd }}$ measure } & $22.4 \pm 3.7$ & 22 & $26.0 \pm 2.4$ & 26 & $23.6 \pm 3.7$ & 24 & 0.015 & $\mathrm{~K}$ \\
\hline \multicolumn{2}{|l|}{$3^{\text {th }}$ measure } & $23.8 \pm 3.5$ & 24 & $26.7 \pm 4.3$ & 26 & $23.7 \pm 3.5$ & 24 & 0.049 & $\mathrm{~K}$ \\
\hline \multicolumn{10}{|c|}{ Systolic Pressure } \\
\hline $1^{\text {st }}$ measure & & $118.9 \pm 10.3$ & 122 & $128.1 \pm 9.8$ & 130 & $127.8 \pm 6.8$ & 129 & 0.010 & $\mathrm{~K}$ \\
\hline $2^{\text {nd }}$ measure & & $127.6 \pm 10.9$ & 132 & $132.9 \pm 9.5$ & 133 & $127.9 \pm 11.5$ & 129 & 0.050 & $\mathrm{~K}$ \\
\hline $3^{\text {th }}$ measure & & $125.9 \pm 10.5$ & 123 & $131.8 \pm 10.5$ & 131 & $123.3 \pm 9.2$ & 122 & 0.414 & $\mathrm{~K}$ \\
\hline Diastolic $P_{I}$ & essure & & & & & & & & \\
\hline $1^{\text {st }}$ measure & & $82.0 \pm 7.0$ & 81 & $87.6 \pm 9.7$ & 90 & $90.4 \pm 9.2$ & 89 & 0.432 & $\mathrm{~K}$ \\
\hline $2^{\text {nd }}$ measure & & $82.3 \pm 9.7$ & 84 & $86.0 \pm 13.4$ & 87 & $84.1 \pm 8.8$ & 85 & 0.095 & $\mathrm{~K}$ \\
\hline $3^{\text {th }}$ measure & & $78.0 \pm 27.1$ & 74 & $84.6 \pm 9.4$ & 82 & $78.5 \pm 6.5$ & 77 & 0.032 & $\mathrm{~K}$ \\
\hline Saturation & & & & & & & & & \\
\hline $1^{\text {st }}$ measure & & $98.1 \pm 1.1$ & 98 & $98.3 \pm 0.7$ & 98 & $98.4 \pm 1.1$ & 98 & 0.691 & $\mathrm{~K}$ \\
\hline $2^{\text {nd }}$ measure & & $98.1 \pm 1.6$ & 99 & $97.9 \pm 1.3$ & 98 & $97.9 \pm 1.0$ & 98 & 0.691 & $\mathrm{~K}$ \\
\hline $3^{\text {th }}$ measure & & $97.4 \pm 1.2$ & 97 & $96.7 \pm 1.6$ & 97 & $97.1 \pm 1.2$ & 97 & 0.471 & $\mathrm{~K}$ \\
\hline Cycle Ergo & eter Test & (m) & & & & & & & \\
\hline $1^{\text {st }}$ measure & & $0.00 \pm 0.00$ & 0.00 & $0.00 \pm 0.00$ & 0.00 & $0.00 \pm 0.00$ & 0.00 & 1.000 & $\mathrm{~K}$ \\
\hline $2^{\text {nd }}$ measure & & $1.73 \pm 0.26$ & 1.64 & $1.79 \pm 0.44$ & 1.71 & $1.68 \pm 0.40$ & 1.58 & 0.628 & $\mathrm{~K}$ \\
\hline $3^{\text {th }}$ measure & & $1.92 \pm 0.29$ & 1.95 & $2.06 \pm 0.39$ & 1.93 & $1.94 \pm 0.45$ & 1.76 & 0.544 & $\mathrm{~K}$ \\
\hline
\end{tabular}

Values are numbers or mean \pm standard deviation or number. K: Kruskal-wallis (Mann-whitney $u$ test) $/ \mathrm{X}^{2} \mathrm{Chi-squared} \mathrm{test/p} \mathrm{>}$ $0.05 / p<0.05$

group, the $3^{\text {rd }}$ measurement diastolic pressure value was significantly higher than the control group and the above threshold group $(p<0.05)$. The $3^{\text {rd }}$ measurement diastolic pressure value did not differ significantly between the control group and the above threshold group $(p>0.05)$ (Table 2).

Saturation value did not differ significantly $(p>0.05)$ in the control group, the sub-threshold group, and the suprathreshold group (Table 2). In the control group, the sub-threshold group, the above threshold group, the $1^{\text {st }}$ measurement, the $2^{\text {nd }}$ measurement, the $3^{\text {rd }}$ measurement performance did not differ significantly ( $p$ $>0.05$ ) (Table 2).

\section{Discussion}

When the effect of non-invasive VNS on physiological parameters such as blood pressure and pulse is examined in the literature, there are conflicting results [18-22]. In addition, there are no studies on the effect of non-invasive VNS on sportive performance. Therefore, this study was planned to investigate the effect of non- 
invasive transauricular VNS on sportive performance and physiological parameters in healthy individuals.

In an animal experiment conducted by Annoni, et al. in 2015, VNS has been shown to relieve the arterial blood pressure and reduce the number of arrhythmia episodes in hypertensive rats [1]. However, although it is an old study, a study by Tiedt and Religa on dogs in 1979 showed that VNS increased coronary flow in dogs [3] Chen, et al. found in a study conducted in 2015 that high-severity invasive VNS tends to facilitate atrial fibrillation, but it tends to inhibit atrial fibrillation without arrhythmogenic risks to moderate VNS. This indicates that the severity of VNS may produce different physiological effects [23-25]. We are of the opinion that the lack of a certain standardization of VNS applied in these studies may explain to some extent the contradictions that arise in the result part of the application. For this reason, in order to ensure standardization, repetitive studies should be organized on each parameter in which the mechanism of action may occur, in which the application doses are tried in detail and the results should be documented.

Steven, et al. stated that VNS is an FDA-approved treatment method in the case of cardiovascular disease, however, they conducted a study with Sprague-Dawley rats in 2016 with the hypothesis that the use of this application in other disease groups would have effects on a healthy cardiovascular system and that negative side effects could be encountered. As a result of the 10-week study, the decrease in action potential time and the increase in transmission rate showed that the beneficial effects of VNS did not require the presence of an existing autonomic imbalance. Similarly, the authors stated at the end of the study that VNS did not induce any significant changes in the ventricular rejection fraction and the spatial distribution of the action potential time, and therefore VNS did not adversely affect cardiac function. From this point of view, the fact that our study is based on performance increase on healthy individuals shows parallelism with this study [26].

Another study conducted by Chen evaluated the effects of VNS on acute effects and life span on chronic hypertensiverats. As a resultof thestudy, VNSsignificantly increased the long-term survival of hypertensive rats and improved autonomic balance by significantly increasing heart rate variability during stimulation in the acute period. This suggests that this is how the mechanism of formation of positive chronic effects occurs [27]. A drug substance experiment conducted by Lee, et al. revealed that stimulation of parasympathetic activity prevents ventricular dysfunction at the onset of heart failure and increases life expectancy [28].

The studies carried out on people have determined their focal points more specifically and are designed to form a preliminary preparation for the studies to be carried out. Beh and Friedman investigated the treatment of acute vestibular migraine with noninvasive VNS in 2019 and showed a mean decrease of $63.3 \%$ in headache intensity and $46.9 \%$ in vertigo intensity due to acute vestibular migraine. "Our study, however, provides preliminary evidence that nVNS can provide rapid relief in vertigo and headache in acute vestibular migraine and supports further randomized, controlled studies on nVNS" [25]. In another study focusing on migraine, Straube, et al. approached the application a little more specifically and compared the effectiveness of different parameters. They shaped the protocol they applied at $1 \mathrm{~Hz}$ and $25 \mathrm{~Hz}$ wavelengths and found that patients in the $1 \mathrm{~Hz}$ group had significantly more reduction in headache days than patients in the $25 \mathrm{~Hz}$ group in 28 days. $29.4 \%$ of the patients in the $1 \mathrm{~Hz}$ group had a $\geq 50 \%$ reduction in headache days, while this rate was limited to $13.3 \%$ in the $25 \mathrm{~Hz}$ group $[23,29]$. Although the sympathetic or parasympathetic activity intensity of the source of pain in the migraine table varies from case to case, it is controversial in the literature. Therefore, it is unlikely to predict the level of effectiveness of VNSs of different wavelengths and intensities. In another human experiment by Clancy, et al., the effects of ear transcutaneous VNS on 48 healthy participants were investigated. At the end of the study, they concluded that tVNS can increase the desired heart rate and reduce sympathetic nerve stimulation in cases characterized by increased sympathetic nerve activity such as heart failure [30].

Traditionally, the therapeutic intensity of VNS is selected to indicate the engagement of the autonomic nervous system (typically through changes in heart rate (HR)) [31]. However, the stimulation parameters used to treat heart diseases are highly variable depending on different models and studies [1]. It is considered that the main reason for obtaining contradictory results is that the duration, severity and duration of treatment sessions were not optimally determined.

In our study, the fact that there was a partially significant result on the parameters evaluated and that no side effects were observed may be due to the short duration of application and the low number of sessions. There are sufficient studies in the literature that increasing the number of sessions by extending the duration of the session as a forecast will give positive results in the chronic period.

\section{Conclusion}

As shown above, there are few studies in the literature where VNS has negative results and it is related to the high level of application severity. Since the reason for the contradictions can be associated with the duration and number of sessions, it is recommended to repeat the study by increasing the duration and number of sessions. 


\section{Authors' Contribution}

Study Conception: SHH, AVÖ, MA; Study Design: SHH, AVÖ, MA; Supervision: SHH, AVÖ, ÇT; Funding: SHH, AVÖ, ÇT, MA; Materials: SHH, ÇT; Data Collection and/or Processing: SHH, BEP, MA, ÇT, AVÖ; Statistical Analysis and/or Data Interpretation: SHH, BEP, MA, AVÖ; Literature Review: SHH, BEP, MA, AVÖ; Manuscript Preparation: $S H H, B E P, M A, A V O ̈$ and Critical Review: SHH, BEP, AVÖ.

\section{Conflict of Interest}

The authors disclosed no conflict of interest during the preparation or publication of this manuscript.

\section{Sources of Support}

The authors disclosed that they did not receive any grant during conduction or writing of this study.

\section{References}

1. Gökçe EN, Cengiz ZP, Erbaş O (2018) Uzun ömrün sırrı: Vagus siniri. İstanbul Bilim Üniversitesi Florence Nightingale Tıp Dergisi 4: 154-165.

2. Tracey KJ (2003) Inhibition of inflammatory cytokine production by cholinergic agonists and vagus nerve stimulation. Google Patents.

3. Hong GS, Zillekens A, Schneiker B, Pantelis D, de Jonge WJ, et al. (2019) Non-invasive transcutaneous auricular vagus nerve stimulation prevents postoperative ileus and endotoxemia in mice. Neurogastroenterol Motil 31: e13501.

4. Liu JJ, Huang N, Lu Y, Zhao M, Yu XJ, et al (2015) Improving vagal activity ameliorates cardiac fibrosis induced by angiotensin II: in vivo and in vitro. Scientific Reports 5: 1-13.

5. Lataro RM, Silva CA, Fazan Jr R, Rossi MA, Prado CM, et al. (2013) Increase in parasympathetic tone by pyridostigmine prevents ventricular dysfunction during the onset of heart failure. Am J Physiol Regul Integr Comp Physiol 305: R908-R916.

6. Dalli J, Colas RA, Arnardottir H, Serhan CN (2017) Vagal regulation of group 3 innate lymphoid cells and the immunoresolvent PCTR1 controls infection resolution. Immunity: 92-105.

7. Penry JK, Dean JC (1990) Prevention of intractable partial seizures by intermittent vagal stimulation in humans: preliminary results. Epilepsia 31: S40-S43.

8. Ben-Menachem E, Revesz D, Simon BJ, Silberstein S (2015) Surgically implanted and non-invasive vagus nerve stimulation: A review of efficacy, safety and tolerability. European Journal of Neurology 22: 1260-1268.

9. Yuan H, Silberstein SD (2016) Vagus nerve and vagus nerve stimulation, a comprehensive review: Part II. Headache: The Journal of Head and Face Pain 56: 259-266.

10. Straube A, Ellrich, J, Eren O, Blum B, Ruscheweyh R (2015) Treatment of chronic migraine with transcutaneous stimulation of the auricular branch of the vagal nerve (auricular t-VNS): A randomized, monocentric clinical trial. J Headache Pain 16: 543.

11. Silberstein SD, Calhoun AH, Lipton RB, Grosberg BM, Cady RK, et al. (2016) Chronic migraine headache prevention with noninvasive vagus nerve stimulation: The EVENT study. Neurology 87: 529-538.
12. Tassorelli C, Grazzi L, de Tommaso M, Pierangeli G, Martelletti $P$, et al. (2018) Noninvasive vagus nerve stimulation as acute therapy for migraine: The randomized PRESTO study. Neurology 91: e364-e373.

13. Beh SC, Friedman DI (2019) Acute vestibular migraine treatment with noninvasive vagus nerve stimulation. Neurology 93: e1715-e1719.

14. Busch V, Zeman F, Heckel A, Menne F, Ellrich J, et al. (2013) The effect of transcutaneous vagus nerve stimulation on pain perception-an experimental study. Brain Stimul 6: 202-209.

15. Möller M, Mehnert J, Schroeder CF, May A (2020) Noninvasive vagus nerve stimulation and the trigeminal autonomic reflex: an fMRI study. Neurology 94: e1085-e1093.

16. Colzato LS, Ritter SM, Steenbergen L (2018) Transcutaneous vagus nerve stimulation (tVNS) enhances divergent thinking. Neuropsychologia 111: 72-76.

17. Oshinsky ML, Murphy AL, Hekierski Jr H, Cooper M, Simon BJ (2014) Noninvasive vagus nerve stimulation as treatment for trigeminal allodynia. Pain 155: 1037-1042.

18. Villani V, Tsakiris M, Azevedo RT (2014) Transcutaneous vagus nerve stimulation improves interoceptive accuracy. Neuropsychologia 134: 107201.

19. Colzato LS, Sellaro R, Beste C (2017) Darwin revisited: The vagus nerve is a causal element in controlling recognition of other's emotions. Cortex 92: 95-102.

20. Antonino D, Teixeira AL, Maia-Lopes PM, Souza MC, Sabino-Carvalho JL, et al. (2017) Non-invasive vagus nerve stimulation acutely improves spontaneous cardiac baroreflex sensitivity in healthy young men: A randomized placebo-controlled trial. Brain Stimul 10: 875-881.

21. Sabino-Carvalho JL, Antonino D, Teixeira AL, Maia-Lopes PM, Murray AR, et al (2017) Non-invasive Vagus Nerve Stimulation Acutely Improves Blood Pressure Control in a Placebo Controlled Study. The FASEB Journal 31: 848-888.

22. Clancy JA, Mary DA, Witte KK, Greenwood JP, Deuchars SA, et al. (2014) Non-invasive vagus nerve stimulation in healthy humans reduces sympathetic nerve activity. Brain Stimul 7: 871-877.

23. Annoni EM, Xie X, Lee SW, Libbus I, KenKnight BH, et al. (2015) Intermittent electrical stimulation of the right cervical vagus nerve in salt-sensitive hypertensive rats: effects on blood pressure, arrhythmias, and ventricular electrophysiology. Physiol Rep 3: e12476.

24. Tiedt N, Religa A (1979) Vagal control of coronary blood flow in dogs. Basic Res Cardiol 74: 267-276.

25. Chen M, Yu L, Liu Q, Jiang $H$, Zhou S (2015) Vagus nerve stimulation: A spear role or a shield role in atrial fibrillation?. Int J Cardiol 198: 115-116.

26. Lee SW, Li Q, Libbus I, Xie X, KenKnight BH, et al. (2016) Chronic cyclic vagus nerve stimulation has beneficial electrophysiological effects on healthy hearts in the absence of autonomic imbalance. Physiol Rep 4: e12786.

27. Annoni EM, Van Helden D, Guo Y, Levac B, Libbus I, et al. (2019) Chronic low-level vagus nerve stimulation improves long-term survival in salt-sensitive hypertensive rats. Front Physiol 10: 25.

28. Yoo PB, Liu H, Hincapie JG, Ruble SB, Hamann JJ, et al. (2016) Modulation of heart rate by temporally patterned vagus nerve stimulation in the anesthetized dog. Physiol Rep 4: e12689. 
29. Xie X, Lee SW, Johnson C, Ippolito J, Ken Knight BH, et al. (2014) Intermittent vagal nerve stimulation alters the electrophysiological properties of atrium in the myocardial infarction rat model. Annu Int Conf IEEE Eng Med Biol Soc 2014: 1575-1578.

30. Li M, Zheng C, Sato T, Kawada T, Sugimachi M, et al. (2004) Vagal nerve stimulation markedly improves long- term survival after chronic heart failure in rats. Circulation 109: $120-124$.

31. Kong SS, Liu JJ, Hwang TC, Yu XJ, Zhao M, et al. (2012) Optimizing the parameters of vagus nerve stimulation by uniform design in rats with acute myocardial infarction. PLoS One 7: e42799. 